\title{
REFERENCES
}

Ablowitz M J, Ramani A and Segur H, A Connection between Nonlinear Evolution Equations and Ordinary Differential Equations of P-type. I, J. Math. Phys. 21 (1980), 715-721.

Ablowitz M J and Segur H, "Solitons and the Inverse Scattering Transform," SIAM, Philadelphia, 1981.

Adler $M$ and van Moerbeke $P$, The Algebraic Integrability of Geodesic Flow on SO(4), Invent. Math. 67 (1982a), 297-331.

Adler $M$ and van Moerbeke P, Kowalewski's Asymptotic Method, Kac-Moody Lie Algebras and Regularization, Comm. Math. Phys. 83 (1982b), 83-106.

Barouch E, McCoy B M and Wu T T, Zero-Field Susceptibility of the TwoDimensional Ising Model Near $T_{c}$, Phys. Rev. Lett. 31 (1973), 1409-1411.

Bechlivanidis C and Van Moerbeke P, The Goryachev-Chaplygin Top and the Toda Lattice, Comm. Math. Phys. 110 (1987), 317-324.

Bluman G W and Cole J D, "Similarity Methods for Differential Equations," Springer, New York, 1974.

Bountis $T$, Segur $H$ and Vivaldi F, Integrable Hamiltonian Systems and the Painlevé Property, Phys. Rev. A25 (1982), 1257-1264.

Bountis T, "Dynamical Systems and Chaos," On the Analytic Strueture of Chaos in Dynamical Systems in Lecture Notes in Physics, Springer-Verlag, Berlin, 1983. ed. by L Garrido

Bruschi M and Ragnisco O, The Hamiltonian Structure of a Complex Version of the Burgers Hierarchy, J. Math. Phys. 26 (1985), 943-945.

Calogero F and Degasperis A, Reduction Technique for Matrix Nonlinear Evolution Equations Solvable by the Spectral Transform, J. Math. Phys. 22 (1981), 23-31.

Chang Y F, Tabor M and Weiss J, Analytic Structure of the Henon-Heiles Hamiltonian in Integrable and Nonintegrable Regimes, J. Math. Phys. 23 (1982), 531-538. 
Chen H H, Lee Y C and Lin J E, On the Direct Construction of the Inverse Scattering Operator of Integrable Nonlinear Hamiltonian Systems, Physica 26D (1987), 165-170.

Clarkson P A, The Painlevé Property and a Partial Differential Equation with an Essential Singularity, Phys. Lett. 109A (1985), 205- 208.

Clarkson P A, McLeod J B, Olver P and Ramani A, Integrability of Klein-Gordon Equations, SIAM J. Math. Anal. 17 (1986a), 798-802.

Cornille H J, Exact Solutions of the Broadwell model in 1+1 Dimensions, J. Phys. A: Math. Gen. 20 (1987), 1973-1988.

Davis H T, "Introduction to Nonlinear Differential and Integral Equations," Dover, New York, 1962.

Dodd $\mathrm{R}$ and Fordy A, On the Integrability of a System of Coupled KdV Equations, Phys. Lett. 89A (1982), 168-170.

Druyma V S, On the Integration of the Cylindrical Kadomtsev-Petviasheili Equation by the Method of the Invers Problem of the Scattering Theory, Soviet Math. Dokl. 27 (1983), 6-8.

Ercolani N and Siggia D, Painlevé Property and Integrability, Phys. Lett $119 \mathrm{~A}$ (1986), 112-116.

Ernst M H, Nonlinear Model-Boltzmann Equations and Exact Solutions, Phys. Rep 78 (1981), 1-171.

Euler N, Leach P G L, Mahomed F M and Steeb W-H, Symmetry Vector Fields and Similarity Solutions of a Nonlinear Field Equation Describing the Relaxation to a Maxwell Distribution, Int. J. Theor. Phys. (in press) (1988).

Euler N, "Proceedings of the Workshop on Finite Dimensional Integrable Nonlinear Dynamical Systems," Discrete Boltzmann Equations and Painlevé Test, World Scientific, Singapore, 1988. ed. by P G L Leach and W-H Steeb

Fokas A S, A Symmetry Approach to Exactly Solvable Evolution Equations, J. Math. Phys. 21 (1980), 1318-1325.

Fokas A S and Ablowitz M J, On a Unified Approach to Transformations and Elementary Solutions of Painlevé Equations, J. Math. Phys. 23 (1982), 2033-2041.

Fokas A S, Leo R A, Martina L and Soliani G, The Scaling Reduction of the 
Three-Wave Resonant System and the Painleve VI Equation, Phys. Lett. 115A (1986), 329-332.

Fuchsteiner B and Fokas A S, Symplectic Structures, their Bäcklund Transformations and Hereditary Symmetries, Physica 4D (1981), 47-66.

Gardner C S, Greene J M, Kruskal M D and Miura R M, Method for Solving the Korteweg-de Vries Equation, Phys. Rev. Lett. 19 (1967), 1095-1097.

Gibbon J D, Radmore P, Tabor M and Wood D, The Painleve Property and Hirota's Method, Stud. Appl. Math. 72 (1985), 39-63.

Gibbon J D and Tabor M, On the One- and Two-Dimensional Toda Lattice and the Painleve Property, J. Math. Phys. 26 (1985), 1956-1960.

Godunov S K and Sultangazin U M, On Discrete Models of the Kinetic Boltzmann Equation, Russian Math. Surveys 24 (1971), 1-55.

Goldstein P and Infeld E, The Zakharov Equations: A Non-Painleve System with Exact $N$ Soliton Solutions, Phys. Lett. 103A (1984), 8-10.

Gravel A, The Painlevé Test, Bëcklund Tramsformation and Solutions of the Reduced Marwell Bloch Equations, J. Phys. A.: Math. Gen. 19 (1986), 479-484.

Grauel $A$ and Steeb W-H, Similarity Solutions of the Euler Equation and the Navier Stokes Equation in Two Space Dimensions, Int. J. Theor. Phys. 24 (1985), 255-265.

Hajee G, The Investigation of Movable Critical Points of Ordinary Differential Equations and Systems of Ordinary Differential Equations Using Formula Manipulation, Memorandem Nr.386 Department of Applied Mathematics Twentc University of Technology 7500 AE Enschede (1982).

Hastings S P and McLeod J B, A Boundary-Value Problem Associated with the Second Painleve Transcendent and the Korteweg-de Vries Equation, Arch. R. Mech. 73 (1980), 31-51.

Hereman W and Van den Bulck E, "Workshop on Finite Dimensional Integrable Nonlinear Dynamical Systems," MACSYMA Program for the Painleve Test of Nonlinear Ordinary and Partial Differential Equations, World Scientific, Singapore, 1988. ed. by P G L Leach and W-H Steeb

Hidekazu I, Non-Integrability of Hénon-Heiles System and a Theorem of Ziglin, Kodai Math. J. 8 (1985), 120-138. 
Hietarinta J, Direet Method for the Search of the Second Invariant, Phys. Rep. 147 (1987), 87-154.

Hille E, "Ordinary Differential Equations in the Complex Domain," John Wiley, New York, 1976.

Hirota R, Exact Solutions of the KdV Equation for Multiple Collisions of Solitons, Phys. Rev. Lett. 27 (1971), 1192-1194.

Hirota R, A New Form of Bäcklund Transformations and its Relation to the Inverse Scattering Problem, Prog. Theor. Phys. 52 (1974), 1498-1512.

Hirota R, "Lecture Notes in Mathematics 515, Bäcklund Transformations," Direct Method of Finding Exact Solutions of Nonlinear Evolution Equations, Springer-Verlag, Berlin, 1976, pp. 40-68. ed. by R M Miura

Hirota $\mathrm{R}$ and Satsuma J, $\boldsymbol{N}$-Soliton Solution of the Korteweg de Vries Equation with Loss of Nonuniformity Terms, Phys. Soc.Jpn. 41 (1976), 2141-2142.

Hlavatý L, Painlevé Analysis of the Calogero-Degasperis-Fokas Equation, Phys. Lett. 113A (1985), 177-178.

Hlavatý L, Test of Resonances in the Painlevé Analysis, Computer Phys. Comm. 42 (1986), 427-433.

Horozov E, On the Non-Integrability of Gross-Neveu Models, Ann. Phys. 174 (1987), 430-441.

Ince E L, “Ordinary Differential Equations," Dover, New York, 1956.

Ito H, Non-integrability of the Henon-Heiles System and a Theorem of Ziglin, Kodai Math. J 8 (1985), 120-138.

Jimbo M, Miwa T, Môri $Y$ and Sato M, Density Matrix of an Impenetrable Bose Gas and the Fifth Painleve Transcendent, Physica 1D (1980), 80-158.

Jimbo M, Kruskal M D and Miwa T, Painlevé Test for the Self Dual Yang Mills Equation, Phys. Lett. 92A (1982), 59-60.

Kamran N, Lamb K G and Shadwick W F, The Local Equivalence Problem for $d^{2} y / d x^{2}=F(x, y, d y / d x)$ and the Painleve transcendents, J. Differential Geometry 22 (1985), 139-150.

Kawamoto S, Construction of Stationary Wave Solutions, J. Phys. Soc. Jpn. 54 (1985), 1701-1709.

Kentwell G W, Nambu Mechanics and Generalized Poisson Bracket, Phys. Lett. 
114A (1986), 55-57.

Konno $\mathrm{K}$ and Tateno H, Duffing's Equation in Complex Times and Chaos, Prog. Theor. Phys. 72 (1984), 1047-1049.

Kovalevskaya S, "A Russian Childhood," Springer-Verlag, New York, Heidelberg, Berlin, 1978.

Kowalewski S, Zur Theorie der Partiellen Differentialgleichungen, Journal für die reine und angewandte Mathematik 80 (1875), 1-32.

Kowalewski S, Sur le Problème de la Rotation d'un Corps Solide Autour d'un Point Fixe, Acta Mathematica 12 (1880), 177-232.

Kowalewski S, Sur une Propriété du Systeme D'équations Differentielles qui Definit la Rotation d'un Corps Solide Autour d'un Point Fixe, Acta Mathematica 14 (1890), 81-93.

Krook M and Wu T T, Formation of Maxwellian Tails, Phys. Rev. Lett. 36 (1976), 1107-1109.

Kus M, Integrals of Motion for the Lorenz System, J. Phys. A: Math. Gen. 16 (1983), L689-L691.

Lakshmanan and Kaliappan P, Lie Transformations of Nonlinear Evolution Equations, and Painleve Forms, J. Math. Phys. 24 (1983), 795-806.

Lakshmanan M and Sahadevan R, Coupled Quartic Anharmonic Oscillators, Painleve Analyois, and Integrability, Phys. Rev. A31 (1985), 861-876.

Lieb $\mathbf{E} \mathbf{H}$ and Linger W, Exact Analysis of an Interaeting Bose Gas: I. The General Solution and the Ground State, Phys. Rev. 130 (1963), 1605-1616.

McCoy B M, Tracy C A and Wu T T, Painlevé Functions of the Third Kind, J. Math. Phys. 18 (1977), 1058-1092.

McCoy B M and Wu T T, The Two-Dimensional Ising Model, Nucl. Phys. B180 (1981), 89-115.

McLeod J B and Olver P J, The Connection Between Partial Differential Equations Soluble by Inverse Seattering and Ordinary Differential Equations of Painlevé Type, SIAM J. Math. Anal. 14 (1983), 488-506.

Nambu Y, Generalized Hamiltonian Dynamics, Phys. Rev. D7 (1973), 24052412.

Newell A C, Tabor M and Zeng Y B, A Unified Approach to Painleve Expansions, 
Physica 29D (1987), 1-68.

Oevel W and Steeb W-H, Painlevé Analysis for a Time-Dependent Kadomtsev-Petviashvili Equation, Phys. Lett. 103A (1984), 239-242.

Pohlmeyer K, Integrable Hamiltonian Systems and Integration Through Quadratic Constraints, Comm. Math. Phys. 46 (1976), 207-221.

Puri S, Painleve Analysis of the Perturbed Sine-Gordon Equation, Phys. Lett. 107 A (1985), 359-361.

Ramani A, Dorizzi B and Grammaticos G, Painleve Conjecture Revisited, Phys. Rev. Lett. 49 (1982), 1539-1541.

Ramani A, Hietarinta J, Dorizzi B and Grammaticos B, Complete Painlevé Analysis for Coupled Quartic Oscillators in N Dimensions, Phys. Lett. 108A (1985), 55-58.

Rand D W and Winternitz P, ODEPAINLEVÉ - A MACSYMA Package for Painlevé Analysis of Ordinary Differential Equations, Comput. Phys. Comm. 42 (1986), 359-383.

Reid J D, Linearization of Homogeneous Riccati Equations, Phys. Lett. 97 A, 309-310.

Rod D L, On an Example of Ziglin in Hamiltonian Dynamics, Conference Proceedings of the Canadian Mathematical Society 8 (1987), 599-608.

Rod D $L$ and Churchill R C, "Proceedings of the Workshop on Finite Dimensional Integrable Nonlinear Dynamical Systems," On the Applicability of Ziglin's Nonintegrability Theorem, World Scientific, Singapore, 1988. ed. by P G L Leach and W-H Steeb

Rogers and Shadwick, "Bäcklund Transformations and their Applications," Academic Press, 1982.

Roy Chowdhury A, Lie Bäcklund Transformation, Painlevé Transcendent for a Korteweg de Vries Equation with Explicit $x$ dependence, J. Phys. A: Math. Gen. 15 (1982), L343-L346.

Satsuma J and Kaup D J, A Bäcklund Transformation for Higher Order Korteweg-de Vries equation, J. Phys. Soc. Jpn. 43 (1977), 692-697.

Shimada I and Nagashima T, A Numerical Approach to Ergodic Problems of Dissipative Dynamical Systems, Prog. Theor. Phys. 61 (1979), 1605-1616. 
Steeb W-H and Strampp W, Diffusion Equations and Lie and Lie-Bäcklund Transformation Groups, Physica $114 A$ (1982), 95.

Steeb W-H, Kunick $A$ and Strampp W, The Rikitake Two-Disc Dynamo System and the Painleve Property, J. Phys. Soc. Jpn. 52 (1983), 2649-2653.

Steeb W-H and Kunick A, Painleve Property of Anharmonic Systems with an External Periodic Field, Phys. Lett. 95A (1983), 269-272.

Steeb W-H, Kloke M and Spieker B M, Nonlinear Schrödinger Equation, Painleve test, Bäellund Transformation and Solutions, J. Phys. A: Math. Gen. 17 (1984), 825-829.

Steeb W-H, On Lie-Bäcklund Vector Fields and the Evolution Equations $\partial^{2} u / \partial x \partial t=f(t)$ and $\partial u / \partial t=\partial^{2} u / \partial x^{2}+f(u)$, J. Math. Phys. (1984), 237-239.

Steeb W-H and Grauel A, Carleman Model and Integrability, Hadronic J. 8 (1985), 187-191.

Steeb W-H and Louw J A, Parametrically Driven Sine Gordon Equation and Painlevé Test, Phys. Lett. 113A (1985), 61-62.

Steeb W-H and Spieker B M, Kadomtsev-Petviashvili Equation with Explicit x and $t$ Dependence, Phys. Rev. A 31 (1985), 1952-1954.

Steeb W-H, Kloke M, Spieker B M and Grensing D, Relativistic Field Equations and the Painleve Test, Z. Phys. C - Particles and Fields 28 (1985a), 241-245.

Steeb W-H, Kloke M, Spieker B M and Kunick A, Integrability of Dynamical Systems and the Singular Point Analysis, Found. Phys. 15 (1985b), 637-666. Steeb W-H, Kloke M, Spieker B M and Grensing D, Soliton Equations, Commutativity and Painlevé Property, Prog. Theor. Phys. 73 (1985c), 344-348.

Steeb W-H, Louw J A, Leach P G L and Mahomed F M, Hamiltonian Systems with Three Degrees of Freedom, Singular Point Analysis and Chaotic Behavior, Phys. Rev. A 33 (1986a), 2131-2133.

Steeb W-H, Louw J A and Villet C M, Maximal One-Dimensional Lyapunov Exponent and Singular-Point Analysis for a Quartic Hamiltonian, Phys. Rev. A 34 (1986b), 3489-3491.

Steeb W-H and Louw J A, Some Remarks on Painleve Test and Integrability, Phys. Scr. 36 (1987), 11-14. 
Steeb W-H and Euler N, Painlevé Test of the McKean and Carleman Models, Lett. Math. Phys. 13 (1987a), 234-236.

Steeb W-H and Euler N, Lie and Lie Bäcklund Vector Fields and Painlevé Test for a Class of Scale Invariant Partial Differential Equations of First Order, Prog. Theor. Phys. 78 (1987b), 214-223.

Steeb W-H, Louw J A and Villet C M, Linearly Coupled Anharmonic Oscillators and Integrability, Austr. J. Phys. 40 (1987b), 587-592.

Steeb W-H, Louw J A and Maritz M F, Singular Point Analysis, Resonances and Yoshida's Theorem, J. Phys. A: Math. Gen. 20 (1987b), 4027-4030.

Steinberg S, Meromorphic Families of Compact Operators, Arch. Rational Mech. Anal. 31 (1969), 214-223.

Strampp W, Bäcklund Transformations for Diffusion Equations, Physica 6D (1982), 113-118.

Strampp W, Bäcklund Transformations and Recursion Operators via Symmetry, J. Phys. Soc. Jpn. 53 (1984), 4129-4137.

Strampp W, Symmetries and the Painlevé Property, Prog. Theor. Phys. 76 (1986), 802-809.

Strampp W and Renner F, "Proceedings of the Workshop on Finite Dimensional Integrable Nonlinear Dynamical Systems," Painlevé Expansion and Similarity, World Scientific, Singapore, 1988. ed. by P G L Leach and W-H Steeb

Tabor $M$ and Weiss J, Analytic Structure of the Lorenz System, Phys. Rev. A 24 (1981), 2157-2167.

Tabor M and Gibbon J D, Aspects of the Painlevé Property for Partial Differential Equiations, Physica 18D (1986), 180-189.

Tajiri $M$ and Kawamoto S, Reduction of $K d V$ and Cylindrical KdV Equation to Painlevé Equations, J. Phys. Soc. Jpn. 51 (1982), 1678-1681.

Tajiri M, Nishitani $T$ and Kawamoto S, Similarity Solutions of the Kadomtsev-Petviashvili Equation, J. Phys. Soc. Jpn. 51 (1982), 2350-2356.

Tajiri M and Hagiwara M, Reduction to the Second Painleve Equation and $N$-Soliton Solutions of the Three-Dimensional Nonlinear Schrödinger Equation, J. Phys. Soc. Jpn. 53 (1984), 1634-1642.

Tamizhmani K M and Sahadevan R, On the Painleve Property of the SO(2,1) 
Invariant Non-Linear o Model, J. Phys. A: Math. General 18 (1985), L1067 -L1070.

Thual $\mathrm{O}$ and Frisch $\mathrm{U}$, "Combustion and Nonlinear Phenomena," Natural

Boundary in the Kuramoto Model., Les Houches, 1985. eds. P. Clavin, B. Larroutvrou and P. Pelce.

van Moerbeke P, "Proceedings of the International Congress of Mathematics," Algebraic Complete Integrability of Hamiltonian Systems and Kac-Moody Lie Algebras, Warszawa, 1983.

$\operatorname{van}$ Moerbeke P, Algebraic Geometrical Methods in Hamiltonian Mechenics, Phil. Trans. R. Soc. Lond. A 315 (1985), 379-390.

van Moerbeke P, Linearizing Completely Integrable Systems on Complex Algebraic Tori, Institut de Mathematique Pure et Appliquee Universite Catholique de Louvain Rapport no 101 (1986), 1-29.

van Moerbeke, "Proceedings of the Workshop on Finite Dimensional Integrable Nonlinear Dynamical Systems," The Geometry of the Painleve Analysis, World Scientific, Singapore, 1988. ed. by P G L Leach and W-H Steeb Ward R S, The Painleve property for the Self-Dual Gauge-Field Equations, Phys. Lett. 102A (1984), 279-282.

Weiss J, Tabor M and Carnevale G, The Painleve Property for Partial Differential Equations, J. Math. Phys. 24 (1983), 522-526.

Weiss J, The Painleve Property for Partial Differential Equations II: Bäeklund Transformation, Lax Pairs, and the Schwarzian Derivative, J. Math. Phys. 24 (1983), 1405-1413.

Weiss J, Bäcklund Transformation and Linearization of the Henon-Heiles System, Phys. Lett. 102A (1984a), 329-331.

Weiss, J., On Classes of Integrable Systems and the Painleve Property, J. Math. Phys. 25 (1984b), 13-24.

Weiss, J., The Sine-Gordon Equations: Complete and Partial Integrability, J. Math. Phys. 25 (1984c), 2226-2235.

Weiss, J., The Painleve Property and Bäcklund Transformations for a Sequence of Boussinesq Equations, J. Math. Phys. 26 (1985), 258-269.

Weiss, J., Modified Equations, Rational Solutions and the Painleve Property for 
the Kadomtsev-Petviashvili and Hirota-Satsuma Equations, J. Math. Phys. 26 (1985), 2175-2180.

Weiss, J., Bäcklund Transformation and the Painlevé Property, J. Math. Phys. 27 (1986), 1293-1305.

Wu T T, McCoy B M, Tracy C A and Barouch E, Spin-Spin Correlations for the Two-Dimensional Ising Model: Exact Theory in the Scaling Region, Phys. Rev. B 13 (1976), 316-322.

Yoshida H, "Proceedings of RIMS Symposium of Non-Linear Systems - Classical Theory and Quantum Theory," Integrability of Generalized Toda Lattice Systems and Singularities in the Complex T-Plane, World Scientific Publishing, 1983. ed. by Jimbo $M$ and Miura $T$

Yoshida H, Necessary Conditions for the Existence of Algebraic First Integrals I: Kowalewski's Exponents, Celestial Mechanics 31 (1983a), 363-379.

Yoshida H, Necessary Condition for the Existence of Algebraic First Integrals II: Condition for Algebraic Integrability, Celestial Mechanics 31 (1983b), 381399.

Yoshida H, "Proceedings of Sixth Kyoto Summer Institute; Chaos and Statistical Mechanics," Self-Similar Natural Boundaries of Non-Integrable Dynamical Systems in the Complex $t$-Plane, Springer Verlag, 1984. ed. by Kuramoto $Y$ Yoshida H, Existence of Exponentially Unstable Periodic Solutions and the NonIntegrability of Homogeneous Hamiltonian Systems, Physica 21D (1986), 163170.

Yoshida H, A Criterion for the Non-existence of an Additional Integral in Hamiltonian Systems with Homogeneous Potential, Physica 29D (1987a), 128-142.

Yoshida H, Non-integrability of a Class of Perturbed Kepler Problems in Two Dimensions, Phys. Lett. A 120 (1987b), 388-390.

Yoshida H, Exponential Instability of Collision Orbit in the Anisotropic Kepler Problem, preprint (1987c).

Yoshida H, "Proceedings of the Workshop on Finite Dimensional Integrable Nonlinear Dynamical Systems," Ziglin Analysis for Proving Nonintegrability of Hamiltonian Systems, World Scientific, Singapore, 1988. ed. by P G L Leach and W-H Steeb 
Yoshida H, Ramani A, Grammaticos B and Hietarinta J, On the Non-integrability of some Generalized Toda Lattices, Physica 144A (1987a), 310-321.

Yoshida H, Grammaticos B and Ramani A., Painlevé Resonances versus Kowalewski Exponents: Some Exact Results on Singularity Structure and Integrability of Dynamical Systems, Acta App. Math. 8 (1987b), 75-103.

Ziglin S L, Branching of Solutions and Nonexistence of First Integrals in Hamiltonian Mechanics: I, Functional Anal. Appl. 16 (1983a), 181-189.

Ziglin S L, Branching of Solutions and Nonexistence of First Integrals in Hamiltonian Mechanics: II, Functional Anal. Appl. 17 (1983b), 6-17. 


\section{Index of Symbols}

$\mathcal{N}: \quad$ Natural numbers

$Z: \quad$ Integers

$Q$ : Rational numbers

$R$ : Real numbers

$C$ : Complex numbers

$[0,1]: \quad$ Unit interval

$D$ : One or several codimensional one subvarieties

$\left\{e_{1}, e_{2}, e_{3}\right\}: \quad$ Standard basis of $R^{3}$

$\dot{u}: \quad=d u / d t$

$H$ : Hamiltonian

$I$ : $\quad$ First integral

$N$ : degrees of freedom (for a Hamiltonian system)

$n$ : number of first order ordinary differential equations of an autonomous system

$U$ : Potential

$L, A$ : Lax pair

g : Monodromy martix

$B$ : $\quad$ Factor bundle

$B_{p}$ : Reduced phase space

$B$ : Banach space

$G$ : Symmetry group

$S: \quad$ Non-characteristic hypersurface

$S: \quad$ Symmetry vector field

$s: \quad$ Similarity variable

$t: \quad$ Time

$t_{1}$ or $z_{1}: \quad$ Pole position

$\tau: \quad=t-t_{1}$

$s n, c n, d n: \quad$ Elliptic functions

H : Hamiltonian density

$\mathcal{L}: \quad$ Lagrangian density

$\phi$ : Analytic functions whose vanishing defines a noncharacteristic hypersurface 
$f \circ g: \quad$ Composition of functions

$(p, q):=\left(p_{1}, \ldots, p_{N}, q_{1}, \ldots, q_{N}\right)$ Canonical coordinates

$O: \quad$ Futher expansions of a series

$T$ : Transpose

det : Determinant

$M: \quad$ Complex analytic symplectic manifold

$\Gamma$ : $\quad$ Phase curve, Riemann surface

tr: Trace

$\wedge$ : Exterior or Grassmann product

$x$ : Cross product

$[$,$] : Commutator$

$\langle$,$\rangle : Scalar product$

$\|\cdot\|: \quad$ Norm

$\{$,$\} : Poisson bracket$ 


\section{Index}

A

Abelian Variety, 63, 77

Action Variables 57

Airy Functions, 9, 149

Algebraic Completely Integrable, 62

$\alpha$-Method, 69

Angle Coordinates 71

Anharmonic Systems 127

ATOMCC, 100

Attractor, 93

B

Bäcklund Transformations, 2, 136, 137, 140, 184, 189-193

Belousov-Zhabotinski Reaction, 27

Bessel Function, 85

Bethe Ansatz, 1

Bifurcation Point, 107

Boltzmann Equation, 281, 301

Boussinesq Equation, 2, 251

Briot-Bouquet Equation, 81

Broadwell Model, 281, 292

Brussel Model, 131

Burgers Equation, 242, 281 
C

Calogero-Degasperis-Fokas Equation, 262

Carleman Model, 281

Cauchy Problem, 6

Cauchy-Riemann Equation, 288

Caudrey-Dodd-Gibbon Equation, 147

Chaotic Behaviour, 1, 3, 91, 93, 109

Cole Hopf Transformation, 243, 294

Completely Integrable, 62, 63

Complex Algebraic Tori, 63

Conservation Laws, 204-207

Conservative Systems, 3, 103

D

Degenerated Resonance, 28

Discrete Boltzmann Equations, 281

Dissipative Systems, 3, 64, 117

Double Sine-Gordon Equation, 279

$\mathbf{E}$

Elliptic Functions, 9, 91

Euler-Poisson System, 3, 157, 158

Euler's Rigid Body, 1

F

Fuchsian Functions, 10

Fundamental Triangle, 97

G

Gateaux Derivative, 184, 185

Gel'fand-Levitan Equation, 169

Generalized Hamiltonian System, 61

Goryachev-Chaplygin Top, 74

Gross-Neveu Model, 164-166 
H

Hamiltonian

Conservative, 3, 56, 103

Generalized, 61

Hénon-Heiles, 94, 103, 143, 157, 160

Quartic, 106

Harry Dym Equation, 208

Hausdorff Dimension, 98

Hénon-Heiles Model, 94, 103, 143, 157, 160

Hessian Matrix, 154

Hirota

Bilinear Technique, 4, 210-234

Hopf Bifurcation, 16

Hyperelliptic Separable Hamiltonian System, 71

I

Integrability, 56

Integrability, Liouville 56

Integrability Coefficient, 154

Integral, First, 56

Inverse Scattering Transform, 1, 213

J

Jordan Canonical Form, 18

K

Kac Moody Algebras, 245

Kadomtsev-Petviashvili Equation, 2, 190-195

Kay and Moses's Expression, 211

Klein-Gordon Equation, 258, 276

Kleinian Functions, 10

Korteweg de Vries Equation, 1, 210, 245 
Kowalewski's

Asymptotic Method, 8

Determinant, 19, 104

Exponent, 16-19

Top, 1

Kuramoto Model, 94, 98

L

Lagrange

Characteristic Equation, 172

Top, 1

Lax

Pair, 61

Representation, 1, 61

Legendre Transformation, 176

Lie Bācklund Vector Fields, 284

Limit-Cycle Systems, 76, 130, 131-134

Liouville

Equation, 259

Integrable, 56

Theorem, 56

Logarithmic Psi Series, 129, 140

Lorenz Model, 66, 92, 117

Lotka-Voltera Model, 65, 124, 137

Lyapunov Exponents, 23, 107

M

MACSYMA, 101, 102

Massless Thirring Model, 176

Maxwell-Bloch Equation, Reduced, 266

McKean Model, 281

Miura Transformation, 208 
Möbius

Transformation, 81, 138, 139

Modified Burgers Equation, 293

Modified Korteweg de Vries Equation, 249

Monodromy

Group, 150, 153

Matrix, 153

$\mathbf{N}$

Nambu Mechanics, 3, 114

Natural Boundary, 98

Normal Variational Equation, 152

Novikov Type Equations, 147

$\mathbf{P}$

Painlevé

Conjecture, 2, 169

Equations, 1, 9, 80

Property, 9, 175

Necessary Condition, 10, 11, 177

Sufficient Condition, 29

Test, 26, 68

Transcendents, 3,80

Periodic Toda Lattice, 1, 12, 60, 109

Psi-Series, 20, 129

$\mathbf{Q}$

Quartic Hamiltonian, 106, 109, 113

Quasi Painlevé Test, 73 
$\mathbf{R}$

Recursion Operator, 187, 294

REDUCE, 101, 102

Reduced Ansatz, 102

Reduced Phase Space, 155

Reduced (or normal) Variational Equation, 22

Resonance, 20

Riemann

Surface, 152

Rikitake Dynamo System, 121

$\mathbf{S}$

Schrödinger Equation (nonlinear), 1, 228, 254

Schwarzian

Derivative, 3, 87, 143

Self-Similar Natural Boundaries, 94

Similarity

Ansatz, 167

Invariant, 64

Variable, 167

Sine-Gordon Equation, 258

Singular Point Analysis, 1, 8

Soliton

Equations, 2, 241

Stationary Point (stable and unstable), 16

Symmetry

Vector Fields, 4, 167, 168, 284, 286 
$\mathbf{T}$

Time-Dependent First Integral, 65

Toda Lattice System, 60, 109

Tori, 62

$\mathbf{U}$

$u^{4}-$ Field Equation, 276

V

Van der Pol Oscillator, 130

Variational Equation, 13, 22, 23, 153, 155

Reduced 22,23

W

Wave Equation, 58

Weak Painlevé Test, 73

Weber-Hermite Functions, 85

Whittaker functions, 85

Y

Yang Mills Theory, 157

$\mathbf{Z}$

Zakharov-Shabat Representation, 193-195

Ziglin's Theorem, 150 